\title{
Development and evaluation of Gappy-POD for noisy PIV measurements in gas turbine combustors
}

\author{
Pankaj Saini* and Adam M. Steinberg ${ }^{\dagger}$ \\ University of Toronto, Toronto, ON, M3H 5T6, Canada
}

Christoph M. Arndt

German Aerospace Center, Pfaffenwaldring 38-40, 70569 Stuttgart, Germany

\begin{abstract}
Gappy proper orthogonal decomposition (GPOD) is assessed here as a data reconstruction technique for particle image velocimetry (PIV) measurements, specifically those applicable to gas turbine combustors (GTC). At practical operating conditions, PIV measurements are plagued with issues, such as low signal-to-noise ratios, that result in significant gaps in data. Four GPOD methods are studied here, including a new method that makes use of a median filter (MF) outlier detection technique to adaptively update reconstructions between iterations. The analysis of the performance of the GPOD methods is done by implementing them on a non-gappy PIV data set. Artificial gaps of varying amounts are added to this non-gappy data set in a manner similar to the gaps found in real experimental data. Additionally, two criteria to check for GPOD convergence are investigated. It was found that the MF method produced the lowest reconstruction error regardless of the amount of gappiness. Furthermore, the MF method was found to be relatively insensitive to the accuracy of the convergence criterion. The MF GPOD therefore is an effective method for filling in missing data in PIV measurements of gas turbine combustor flows.
\end{abstract}

\section{List of Symbols}

$N \quad$ Total number of snapshots in a data set

$P \quad$ Number of POD/GPOD modes

$a_{i}, b_{i} \quad$ POD temporal coefficients

$\phi_{i} \quad$ POD spatial eigenmodes

$\boldsymbol{R} \quad$ Correlation matrix

$\lambda_{n, j, i} \quad$ POD eigenvalues

$\vec{u} \quad$ Velocity

$\bar{\xi}_{n, j} \quad$ Approximate filled field

$\breve{\bar{\xi}}_{n, j} \quad$ POD approximation of field

$\hat{\xi}_{n} \quad$ GPOD reconstructed field at the end of main iteration $n$ (ES and Gunes methods)

$\hat{\hat{\xi}}_{n} \quad$ GPOD reconstructed field at the end of main iteration $n$ (FS and MF methods)

$\tilde{\xi}_{n, j} \quad$ POD approximation of field using coefficients $b_{i}$

$F_{s, n} \quad$ Field smoothness parameter (FS method)

\footnotetext{
* Graduate Student, Institute of Aerospace Studies, Student Member AIAA

${ }^{\dagger}$ Assistant Professor, Institute of Aerospace Studies, Senior Member AIAA

${ }^{\ddagger}$ Research Associate, Institute of Combustion Technology
} 


$\begin{array}{ll}R_{n} & \text { Normalized residual parameter (MF method) } \\ \epsilon & \text { Minimum normalization level (MF method) } \\ \vec{r}_{\vec{x}_{c p}, t_{k}} & \text { Residuals of vectors adjacent to } \vec{x}_{c[]} \\ \varepsilon & \text { Convergence tolerance for sub iterations } \\ E & \text { Reconstruction error over entire data set } \\ \psi & \text { Reconstruction error over a single snapshot } \\ K_{P_{n}} & \text { Kinetic energy calculated from POD eigenvalues } \\ G & \text { Gappiness percentage } \\ D_{n} & \text { Nozzle diameter } \\ q & \text { Turbulent kinetic energy calculated at center of jet } \\ \dot{m}_{A} & \text { Air flow rate (g/min) } \\ \dot{m}_{C H 4} & \text { Fuel flow rate (g/min) } \\ P_{t h} & \text { Thermal power }(\mathrm{kW}) \\ P^{*} & \text { Main iteration }\left(\mathrm{GPOD} \text { mode) at which } E=E^{*}\right. \\ E^{*} & \text { Minimum reconstruction error } \\ V_{m a g} & \text { Velocity magnitude }\end{array}$

\section{Subscripts}

$\begin{array}{ll}n & \text { GPOD main iteration } \\ j & \text { GPOD sub iteration } \\ d & \text { Valid data } \\ g & \text { 'Real' gaps } \\ c c & \text { Convergence checking gaps } \\ i & \text { Index of POD modes } \\ c p & \text { Center point } \\ a d & \text { Points adjacent to center point } c p \\ s & \text { Index of vectors adjacent to center point } c p\end{array}$

\section{Introduction}

Designing gas turbine combustors (GTC) is a challenging task, given the interaction of the combustion chemistry, acoustics, etc. with the turbulent, multi-phase flows. There is a need to better understand these interactions in order to validate computational fluid dynamics (CFD) simulations and help improve the combustor design. For instance, in the case of lean premixed (LP) combustors, issues such as thermoacoustic instabilities can affect performance and even lead to catastrophic structural damage. ${ }^{1}$ These instabilities are a result of pressure fluctuations that cause variations in the heat release rate, which further perturbs the flow field. ${ }^{1}$ In order to better elucidate the physics behind this thermoacoustic coupling, it is necessary to understand the coupled flame and flow dynamics at engine relevant conditions; the latter of two can be achieved through velocity measurements. However, getting accurate velocity 
field measurements in GTCs can be challenging, particularly for temporally resolved measurements at practical operating conditions.

One of the most common velocity measurement techniques in GTCs is particle image velocimetry (PIV). However, when run in high pressure flames similar to ones typically found in GTCs, PIV measurements can be plagued with various issues. Poor signal to noise ratios can result from the use of lasers with low pulse energies, such as those running at multi-kHz repetition rate, and from background noise caused by soot and flame luminescence. Additionally, non-uniform particle seeding can result in voids in the particle field. All these factors culminate to significant gaps in the processed velocity fields which can hinder the analysis of the data. The goal of this paper is to evaluate a method for finding high accuracy estimates for gaps in the data using gappy proper orthogonal decomposition (GPOD).

More specifically, the goal is to fill in the gaps using information from the complete time series of measurements, as opposed to just the local spatial information. It is possible to fill gaps with estimates from spatially interpolating techniques, such as Kriging interpolation, but Gunes et. $\mathrm{ll}^{2}$ found that this technique was most effective for data sets with low temporal resolution. Given that most PIV data sets contain hundreds of snapshots of the velocity field (thousands for experiments run at a multi-kHz repetition rate), it is possible to use the high temporal resolution of the data sets to calculate a better estimate for the data within the gaps. Gappy proper orthogonal decomposition (GPOD) is one such data reconstruction technique that works on this principle.

The primary objective of this paper is to apply and analyze the performance of GPOD techniques on the turbulent swirl flames typically found in modern gas turbine combustors, something that has not previously been done to the authors' knowledge. GPOD is applied on a high-quality atmospheric pressure PIV data set with artificial gaps added, in varying amounts, to the data set in order to mimic noise found in high pressure data. Four different GPOD methods are implemented in this paper: three adapted from literature and a new method that utilizes a PIV post-processing tool. ${ }^{3,2,4,5}$ Section II describes the GPOD methods and their implementation, Section III describes the experimental data on which GPOD is implemented, and Section IV presents the results of the analysis.

\section{Methodology}

\section{A. Proper Orthogonal Decomposition}

POD is a data analysis method that is used to get low dimensional approximations of high-dimensional processes. This is done through a linear combination of basis functions that are calculated from the data directly. POD provides the most efficient way of capturing the dominant components in the data, which it does by constructing the approximation using only the most energetic modes. ${ }^{6}$ Additionally, no prior knowledge is required regarding the process that generates the data. These features make POD quite apt for studying turbulent flows. ${ }^{7}$ In this work, the method of snapshots described by ${ }^{7}$ is used.

Given a set of $N$ measurements of the field $\xi(\vec{x}, t)$, its POD approximation using $P$ modes $\left(\breve{\xi}_{P}\right)$ is a linear combination of $P$ temporal coefficients $a_{i}$ and spatial modes $\phi_{i}$

$$
\breve{\bar{\xi}}_{P}\left(\vec{x}, t_{k}\right)=\sum_{i=1}^{P} a_{i}^{k} \phi_{i}(\vec{x}) ; \quad k \in[1, N]
$$

Typically, the number of modes used for reconstruction is much fewer than the total number of snapshots of $\xi(P \ll N)$. The spatial modes are defined as a linear combination of the temporal coefficients and the snapshots

$$
\phi_{i}(\vec{x})=\sum_{k=1}^{N} a_{i}^{k} \xi\left(\vec{x}, t_{k}\right)
$$

The coefficients, $a_{i}$, are found by solving for the eigenvectors of the correlation matrix $\vec{R}$

$$
\begin{aligned}
& R_{i j}=\frac{\left(\xi\left(\vec{x}, t_{i}\right), \xi\left(\vec{x}, t_{j}\right)\right)}{N} \\
& \boldsymbol{R} \boldsymbol{a}=\boldsymbol{\Lambda} \boldsymbol{a}
\end{aligned}
$$


In the context of velocity fields, the eigenvalues of the correlation matrix, $\lambda_{i}$ (elements of the diagonal matrix $\boldsymbol{\Lambda}$ ), correspond to the energy of the flow contained in mode $i$. These eigenvalues are ordered such that $\lambda_{1}>\lambda_{2}>\lambda_{3} \ldots$

\section{B. GPOD Methods}

GPOD is an iterative implementation of POD that can be used to get approximations for gaps in measurements. Initially, the gaps in the data set are filled with the temporal mean at each point, in order to prevent POD from attempting to recreate zeros at the gaps. A POD approximation of the field is then calculated, using a particular number of modes, and the result is used to get an approximation of the data in the gaps. The resulting field is passed onto the next iteration and this process is repeated until a certain convergence criterion is satisfied.

The capabilities of the GPOD methods are assessed here by artificially adding gaps to a high quality PIV data set, implementing GPOD on those gappy fields, and then comparing the resulting reconstructions to the original non-gappy fields. The high quality data set, which is described in Section III, has essentially no missing vectors and so is appropriate for this type of assessment. The main parameter considered when adding gaps to the data is the gappiness percentage $(G)$, which is defined as the ratio of the number of missing vectors (gaps) to the total number of possible vector points. In this work, $G$ was set to $15 \%, 25 \%$, and $50 \%$. In order to mimic the distribution of real gaps in time, the total number of missing vectors was distributed in a Gaussian manner across snapshots, with the means of the distributions equaling the aforementioned values.

Before introducing the GPOD methods, a discussion of the number of POD modes used in the reconstructions, $P$, is necessary. For a poor initial guess, a high value of $P$ will result in the POD approximation recreating fine details of that poor guess. If $P$ is set too low, then not enough details will be recreated and consequently the reconstruction accuracy will be lower. In order to find the optimal number of modes, Venturi et. $\mathrm{al}^{8}$ introduced an iterative implementation of GPOD, wherein the number of modes, $P_{n}$, is increased incrementally. The initial number of modes is kept low, typically around $P_{0}=2$, and the modes are incremented by 1 , with the reconstructed field from one mode being passed as the initial field for the next one. This iterative procedure has been shown to improve the accuracy of GPOD significantly. ${ }^{8,2}$ Implementing this procedure results in two sets of iterations, ones on the POD convergence with a given number of modes (sub-iterations) and ones on the number of modes used (main-iterations).

Four GPOD methods are implemented here: two underlying methods introduced by Sirovich et. $\mathrm{al}^{3}$ and Gunes et. al, ${ }^{2}$ and two adaptive methods that utilize an outlier detection method between main iterations. The first two methods are classified in this work as non-adaptive methods, since the updated approximation for each gap is accepted regardless of any metric. The adaptive methods, on the other hand, utilize an outlier detection method to potentially reduce reconstruction error by allowing each missing data point to use the optimal number of modes in its approximation. The first adaptive method, introduced by Raben et. $\mathrm{al}^{4}$ uses field smoothness as the outlier detection technique, while the other, a new method, uses median filtering. Furthermore, GPOD is performed on each velocity component $\left(u_{x}, u_{y}, u_{z}\right)$ separately, in order to allow for independent convergence.

\section{Everson Sirovich Method}

The Everson-Sirovich (ES) method was the first GPOD method to be introduced; ${ }^{3}$ its procedure is as follows:

1. For a given main iteration $(n)$, gaps in the original gappy field $(\xi)$ are filled with an initial guess, resulting in a approximate filled field $\left(\bar{\xi}_{n, 0}\right)$. For $n=0$, the guess at a given spatial point is based on the mean at that point, viz.

$$
\bar{\xi}_{0,0}\left(\vec{x}, t_{k}\right)= \begin{cases}\xi\left(\vec{x}, t_{k}\right) & \left.\vec{x} \in \vec{x}_{d}\right|_{t_{k}} \\ \langle\xi(\vec{x})\rangle & \left.\vec{x} \in \vec{x}_{g}\right|_{t_{k}}\end{cases}
$$

where $\langle\xi(\vec{x})\rangle$ is the temporal mean of $\xi$ only over times with valid data at the location being evaluated. For $n>0, \bar{\xi}_{n, 0}$ is the output from previous main iteration, i.e. $\bar{\xi}_{n, 0}=\hat{\xi}_{n-1}$ (see Step 5). 
2. POD analysis is performed on $\bar{\xi}_{n, j}$ using $P_{n}$ modes, resulting in a POD approximation

$$
\breve{\bar{\xi}}_{n, j}\left(\vec{x}, t_{k}\right)=\sum_{i=1}^{P_{n}} a_{i}^{k} \phi_{i}(\vec{x})
$$

3. A second set of temporal coefficients $\left(b_{i}\right)$ is calculated by minimizing the error between the known data and an approximation based on the POD spatial modes, i.e. minimizing

$$
\sum_{k=1}^{N}\left\|\xi\left(\left.\vec{x}_{d}\right|_{t_{k}}, t_{k}\right)-\tilde{\xi}_{n, j}\left(\left.\vec{x}_{d}\right|_{t_{k}}, t_{k}\right)\right\|
$$

where $\tilde{\xi}_{n, j}\left(\vec{x}, t_{k}\right)=\sum_{i=1}^{P} b_{i}^{k} \phi_{i}(\vec{x})$.

4. The gaps in the original data are filled using the POD-like reconstruction arising from the minimization problem

$$
\bar{\xi}_{n, j+1}\left(\vec{x}, t_{k}\right)= \begin{cases}\xi\left(\vec{x}, t_{k}\right) & \left.\vec{x} \in \vec{x}_{d}\right|_{t_{k}} \\ \tilde{\xi}_{n, j}\left(\vec{x}, t_{k}\right) & \left.\vec{x} \in \vec{x}_{g}\right|_{t_{k}}\end{cases}
$$

5. Steps (2-4) are repeated with $j=j+1$ until the POD eigenvalues calculated in step 2 converge within some preset tolerance, at which point the final approximation at main iteration $n$ is stored as $\hat{\xi}_{n}$, and passed to the next main-iteration.

While solving the minimization problem increases the accuracy of the estimate in the gaps, during each sub-iteration, it also increases the computational cost of the method.

\section{Gunes Method}

The method introduced by Gunes et. $\mathrm{al}^{2}$ is similar to the ES method, except that it uses $a_{i}$ directly to estimate the gaps in the data, i.e. $\tilde{\xi}=\breve{\bar{\xi}}$. There is no minimization problem to solve, and so the Gunes method is computationally more efficient. However, the accuracy is lower for a given value of $P$, but this decrease was found to be small when both the methods were implemented in the aforementioned iterative approach (with respect to the modes). ${ }^{2,4}$

\section{Field Smoothness Method}

The field smoothness (FS) method implemented here has been adapted from the adaptive reconstruction POD (ARPOD) method introduced by Raben et. al. ${ }^{4}$ Both methods make use of an outlier detection method based on field smoothness that is applied between the main iterations. The individual points are only updated with the new approximation value if that value is smoother than the previous approximation. For a point $\vec{x}_{c p}$ in a $2 \mathrm{D}$ field, the field smoothness is defined as

$$
\begin{aligned}
& \left.F_{s}(\xi)\right|_{\vec{x}_{c p}, t_{k}}= \\
& \left.\frac{\partial^{2} \xi}{\partial x^{2}}\right|_{\vec{x}_{c p}, t_{k}} ^{2}+\left.2 \frac{\partial^{2} \xi}{\partial x \partial y}\right|_{\vec{x}_{c p}, t_{k}} ^{2}+\left.\frac{\partial^{2} \xi}{\partial y^{2}}\right|_{\vec{x}_{c p}, t_{k}} ^{2}
\end{aligned}
$$

Here, the FS method is run with a Gunes method core. That is,

1. Gunes method is run with $P_{n}$ modes on a field $\bar{\xi}_{n, 0}$ to get an approximation $\hat{\xi}_{n}$.

2. For $n>0$, the field smoothness is calculated for the previous and current reconstructions, $F_{s, n-1}=$ $F_{s}\left(\hat{\xi}_{n-1}\right)$ and $F_{s, n}=F_{s}\left(\hat{\xi}_{n}\right)$ respectively.

3. The input to the next main iteration is is updated such that

$$
\begin{aligned}
& \hat{\hat{\xi}}_{n}\left(\vec{x}, t_{k}\right)= \\
& \begin{cases}\hat{\xi}_{n-1}\left(\vec{x}_{c p}, t_{k}\right), & \text { if }\left.F_{s, n-1}\right|_{x_{c p}, t_{k}} \leq\left. F_{s, n}\right|_{\vec{x}_{c p}, t_{k}} \\
\hat{\xi}_{n}\left(\vec{x}_{c p}, t_{k}\right), & \text { if }\left.F_{s, n-1}\right|_{\vec{x}_{c p}, t_{k}}>\left.F_{s, n}\right|_{\vec{x}_{c p}, t_{k}}\end{cases}
\end{aligned}
$$

and $\bar{\xi}_{n+1,0}=\hat{\hat{\xi}}_{n}$. 
4. Steps (1-3) are repeated with increasing modes $(n=n+1)$ until the main iteration convergence criterion is reached.

The difference between the FS method and the ARPOD method is in the way $\hat{\xi}_{n+1}$ is updated. In this work, a single pass approach of the FS calculation is used, whereas ARPOD makes use a multi-pass iterative approach. The single pass approach was employed here because Raben et. $\mathrm{al}^{4}$ found that doing so works better for cases in which missing data are grouped in clusters, as is the case here.

\section{Median Filter Method}

The median filter (MF) GPOD method introduced here also makes use of an outlier detection technique between the main iterations. However, instead of field smoothness the normalized median filter of Westerweel et. $\mathrm{al}^{5}$ is used. This outlier detection technique was noted for having a high detection efficiency and being simple to automate, when compared to other techniques. ${ }^{9}$ It requires the calculation of the normalized residual of $\xi$ at point $\vec{x}_{c p}$ centered in a neighborhood of adjacent vectors $\left(\vec{x}_{c, a d}\right)$, viz.

$$
\left.R(\xi)\right|_{\vec{x}_{c p}, t_{k}}=\frac{\mid \xi\left(\vec{x}_{c p}, t_{k}\right)-\text { median }\left(\xi\left(\vec{x}_{c, a d}, t_{k}\right)\right) \mid}{\text { median }\left(\left.\vec{r}_{a d}\right|_{\vec{x}_{c p}, t_{k}}\right)+\epsilon}
$$

The first term in the denominator is the median of the residuals of the vectors adjacent to $\vec{x}_{c p}$, and is defined as $\left.\vec{r}_{a d}\right|_{\vec{x}_{c p}, t_{k}}=\left\{\left.r_{1}\right|_{\vec{x}_{c p}, t_{k}},\left.r_{2}\right|_{\vec{x}_{c p}, t_{k}}, \ldots\right\}$, where each residual is

$$
\left.r_{s}\right|_{\vec{x}_{c p}, t_{k}}=\left|\xi\left(\vec{x}_{s}, t_{k}\right)-\operatorname{median}\left(\xi\left(\vec{x}_{c, a d}, t_{k}\right)\right)\right| ; \quad \vec{x}_{s} \in \vec{x}_{a d}
$$

For cases where median $\left(\left.\vec{r}_{a d}\right|_{\vec{x}_{c p}, t_{k}}\right)$ is close to zero, such as in uniform flows, a minimum normalization level $(\epsilon)$ is added to the denominator. A value of $\epsilon=0.1$ typically is used and so is employed here. Additionally, the size of the neighbourhood was kept at 9 (i.e. 1 center vector and 8 adjacent vectors). This leads to the following procedure for the MF method:

1. Gunes method is run with $P_{n}$ modes on a field $\bar{\xi}_{n, 0}$ to get an approximation $\hat{\xi}_{n}$.

2. For $n>0$, the normalized median residual is calculated for the previous and current reconstructions: $R_{n-1}=R\left(\hat{\xi}_{n-1}\right)$ and $R_{n}=R\left(\hat{\xi}_{n}\right)$ respectively.

3. The input to the next main iteration is is updated such that

$$
\begin{aligned}
& \hat{\hat{\xi}}_{n}\left(\vec{x}, t_{k}\right)= \\
& \begin{cases}\hat{\xi}_{n-1}\left(\vec{x}_{c p}, t_{k}\right), & \text { if }\left.R_{n-1}\right|_{\vec{x}_{c p}, t_{k}} \leq\left. R_{n}\right|_{\vec{x}_{c p}, t_{k}} \\
\hat{\xi}_{n}\left(\vec{x}_{c p}, t_{k}\right), & \text { if }\left.R_{n-1}\right|_{\vec{x}_{c p}, t_{k}}>\left.R_{n}\right|_{\vec{x}_{c p}, t_{k}}\end{cases}
\end{aligned}
$$

and $\bar{\xi}_{n+1,0}=\hat{\hat{\xi}}_{n}$.

4. Steps (1-3) are repeated with increasing modes $(n=n+1)$ until the main iteration convergence criterion is reached.

\section{Convergence Criteria}

Given the iterative implementation of GPOD, two convergence criteria are required: one for the main iterations and one for the sub-iterations. The convergence for the sub-iterations is based on the convergence of the eigenvalues associated with the POD calculation, $\lambda_{n, j, i}$, where the third index $(i)$ indicates the POD mode associated with that eigenvalue. Specifically, convergence was based on

$$
\frac{1}{P_{n}} \sum_{i=1}^{P_{n}}\left|\frac{\lambda_{n, j, i}-\lambda_{n, j-1, i}}{\lambda_{n, j, i}}\right|<\varepsilon
$$

It was determined that a value of $\varepsilon=0.1$ produced the lowest error.

Choosing a suitable convergence criterion for the main iterations is important as the reconstruction error does not monotonically decrease with $P .^{8,4,2}$ The reconstruction error using a particular number of modes is defined as

$$
\begin{aligned}
E\left(P_{n}\right) & =\frac{1}{N} \sum_{k=1}^{N} \psi_{k}\left(P_{n}\right) \\
\psi_{k}\left(P_{n}\right) & =\frac{\left\|\hat{\xi}_{n}\left(\left.\vec{x}_{g}\right|_{t_{k}}, t_{k}\right)-\xi\left(\left.\vec{x}_{g}\right|_{t_{k}}, t_{k}\right)\right\|}{\left\|\xi\left(\left.\vec{x}_{g}\right|_{t_{k}}, t_{k}\right)\right\|}
\end{aligned}
$$


where $\|$.$\| is the matrix 2-norm. This error equation is similar to the time averaged relative error$ used by Gunes et. al. ${ }^{2}$ Calculating this error requires knowledge of the true data in the gaps, which is not available for real gappy data, but for this analysis it can be calculated since the gaps have been artificially added to the complete data sets. This reconstruction error is used here to evaluate different convergence criteria for the main iterations based on how well they identify the number of modes at which $E$ is minimized.

Two different convergence criteria for the main iterations are tested here. The first one was introduced by Gunes et. $\mathrm{al}^{2}$ and it consists of adding additional convergence checking (CC) gaps to the already gappy data. In order to reduce their influence on the accuracy of the GPOD reconstruction of the real gaps, the amount of CC gaps is kept low, around $3 \%$ of the data. Since the CC gaps are artificial, the reconstruction error over these gaps $\left(E_{c c}\right)$ can be calculated even for real gappy data. Convergence is achieved when $E_{c c}\left(P_{n}\right)$ is minimized.

The second converge checking technique consists of analyzing the kinetic energy $(K)$ of the POD reconstruction, as expressed by the eigenvalues. This technique was proposed by Venturi et. al, ${ }^{8}$ and it states that the optimal number of modes is one that satisfies

$$
\left|K_{P_{n}}-K_{P_{0}}\right| \text { is a maximum, where } K_{P_{n}}=\sum_{i=1}^{N} \lambda_{n, j, i}^{2}
$$

It should be noted that these eigenvalues are calculated for all the modes $(1 . . N)$, not just the $P_{n}$ modes used in the reconstruction.

\section{Experimental Data}

A high quality PIV data set taken from a turbulent swirl flame in an atmospheric pressure gas turbine model combustor is used to test the capabilities of GPOD. The combustor, shown in Fig. 1, and the data used here were previously described by Steinberg et al. ${ }^{10}$ and Caux-Brisebois. ${ }^{11}$ In the experiment, fuel $\left(\mathrm{CH}_{4}\right)$ and air were mixed in a commercial premixer, fed through a plenum (78 $\mathrm{mm}$ diameter, $100 \mathrm{~mm}$ length) to a radial swirler (12 swirl vanes), before entering the combustion chamber through a nozzle (diameter $D_{n}=17.85 \mathrm{~mm}$ ) with a conical center body. The combustion chamber was $85 \times$ $85 \times 114 \mathrm{~mm}$, ending in a conical contraction to an exhaust tube. Air and fuel mass flow rates were $1024 \mathrm{~g} / \mathrm{min}$ and $42 \mathrm{~g} / \mathrm{min}$, respectively, and the resulting equivalence ratio was 0.70 .

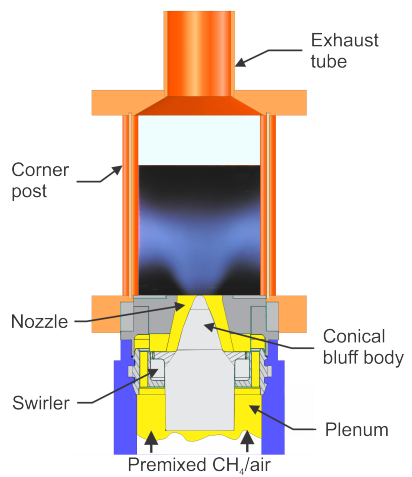

Figure 1: Schematic of model GTC

The stereoscopic PIV (S-PIV) system consisted of a high-repetition-rate Nd:YAG laser (Edgewave, IS-6IIDE) and a pair of high-speed CMOS cameras (LaVision HSS 8) in forward scatter configuration. Laser pulse pairs (532 nm, $2.6 \mathrm{~mJ} / \mathrm{pulse}$, $14 \mathrm{~ns}$ pulse duration, $5-15 \mu$ s between pulses depending on conditions) repeating at $10 \mathrm{kHz}$ were expanded into a sheet having a usable height of about $40 \mathrm{~mm}$ and a full-width-at-half-maximum (FWHM) of approximately $1 \mathrm{~mm}$ at the measurement area. Particle-scattered light from the $\mathrm{TiO}_{2}$ seed was collected into the cameras using $100 \mathrm{~mm}$ focal length commercial camera lenses (Tokina) set to f/5.6. Standard corrections were made for image defocusing and perspective distortion using Scheimpflug adapters and a 3D dot target (LaVision Type 7). Vector fields were computed from the particle image spatial cross-correlation using the LaVision DaVis 8.0 software package, with a final interrogation box size and vector spacing of approximately $1.0 \mathrm{~mm}$ and $0.5 \mathrm{~mm}$ respectively.

The field-of-view imaged by the PIV system was approximately $49 \mathrm{~mm}$ x $38 \mathrm{~mm}$ but, due to optical access restrictions caused by the combustion chamber posts, the S-PIV field-of-view was offset from the burner's centerline, covering the range $x=-17$ to $32 \mathrm{~mm}$. Additionally, reflections from the windows caused increased measurement noise at some locations for $x<0$, so only the measurements at $x>0$ are used. 


\section{Results and Discussion}

In this section, GPOD is evaluated by comparing the minimum absolute reconstruction error $(E)$ of the different GPOD methods. However, as mentioned before, this error cannot be calculated for real gappy data. Therefore, the influence of main iteration convergence criterion on the minimum absolute error will also be analyzed here.

Before beginning the evaluation, the vector fields produced at the different stages of the GPOD process are shown to illustrate the GPOD reconstruction process. A non-gappy vector field at an arbitrary time in the data set is shown in Fig. 2a, while the same field with $G=45 \%$ data gaps added is shown in Fig. 2b. The initial guess to the filled field is shown in Fig. 2c, where the gaps are filled with the temporal mean, and then the MF GPOD method is run on this field. An intermediate solution after $P=10$ main-iterations is shown in Fig. 2d. After several iterations, the final vector field that corresponds to the optimally reconstructed field, i.e. one with the minimum value of $E$, is achieved (Fig. 2e). This field closely resembles the 'real' target vector field.

\section{A. Error Comparison}

Figure 3 contains the curves of the absolute reconstruction error, $E$, for each velocity component, with $G=15 \%$. As $P_{n}$ increases, the error decreases initially, reaches a minimum $\left(E^{*}\right)$, after which it begins to increase monotonically. The curves for all three components exhibit this trend. However, the error minima occur at different modes $\left(P^{*}\right)$ for each velocity component, which indicates that performing GPOD on each velocity component independently is necessary for lowering the total error. The minimum reconstruction error is similar for $u_{x}$ and $u_{y}$ but is higher for $u_{z}$, which is likely a result of higher uncertainty in the $u_{z}$ measurement that is inherent to S-PIV. Given the similarity in the trends for all components, the discussion below will focus only on the $u_{x}$ results.

For the ES and Gunes methods, the error curves, shown in Figure 3, are nearly identical, which is in agreement with the results from Raben et. $\mathrm{al}^{4}$ and Gunes et. al. ${ }^{2}$ This similarity is most likely a result of the iterative implementation of the two methods, with respect to the number of modes. Hence, only results from the Gunes method will be reported from here on. As expected, the adaptive methods have considerably lower error than the non-adaptive ones, with the $E_{F S}^{*}$ and $E_{M F}^{*}$ being around $20 \%$ and $30 \%$ lower than $E_{\text {Gunes }}^{*}$, respectively. The MF method has the lowest error of all the GPOD methods.

Figure 4 shows $E^{*}$ as a function of three different gappiness percentages. All three GPOD methods have the same general trend: an increase in gappiness results in an increase in error. This is because fields with more gaps have less real information passed to the POD, and consequently less accurate GPOD reconstructions. The MF method is the most accurate at all values of $G$, followed by the FS method and the Gunes method.

\section{B. Convergence Criteria for Real Gappy Data}

The analysis presented thus far assumes prior knowledge of the vectors in the gaps in the fields. While this is important in gauging the accuracy of the GPOD methods (via the calculation of $E$ ) and consequently their convergence, it is obviously not applicable to real gappy data. As mentioned in Section $\mathrm{C}$, there are two ways of checking convergence on real gappy data: one way is by introducing artificial $\mathrm{CC}$ gaps and the second is by checking the turbulent kinetic energy $(K)$ calculated from the POD modes. Both these techniques were applied for a non-adaptive method (Gunes) and an adaptive method (MF).

Figure 5a shows the curves of $E$ as a function of the modes and increasing gappiness percentages of the CC gaps. It should be noted that $E$ is calculated only over the 'real' gaps, and not the CC gaps. The GPOD method in this case is the Gunes method, the gappiness percentage of the 'real' gaps is $25 \%$, and curves are presented only for $u_{x}$. Each error curve has two dots on it: a black dot representing the curve's minimum $\left(E^{*}=E\left(P^{*}\right)\right)$ and a white dot representing the mode at which $E_{c c}$, the error over the CC gaps, is a minimum $\left(E_{c c}^{*}=E_{c}\left(P_{c c}^{*}\right)\right)$.

The baseline case, with $3 \%$ gappiness of the convergence checking gaps, shows a large difference in $P^{*}$ and $P_{c}^{*}$, which translates to a large difference in $E^{*}$ and $E_{c c}^{*}$. Therefore, if $P_{c c}^{*}$ was used as a convergence check, the reconstruction error would be quite high. This vast discrepancy is due to the 


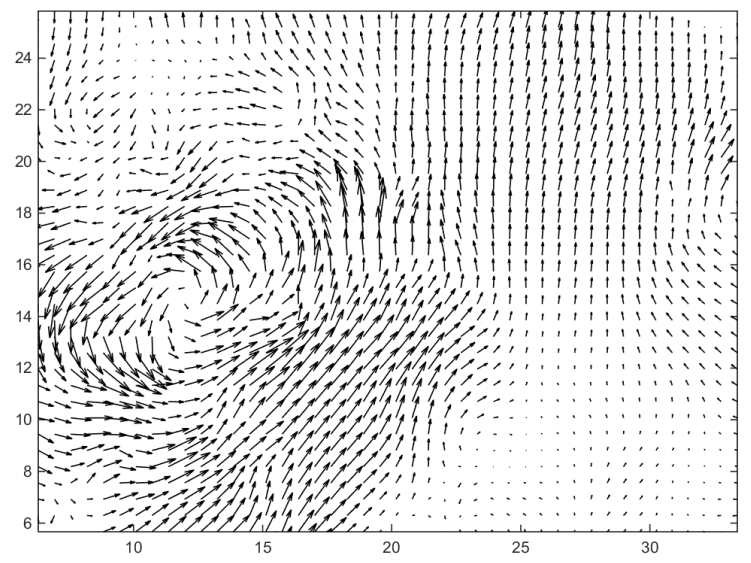

(a) Original field

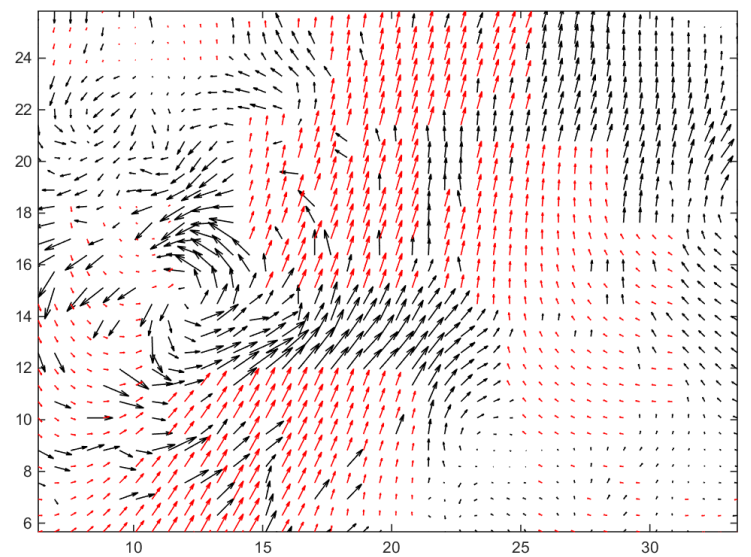

(c) Initial guess (time averaged values)

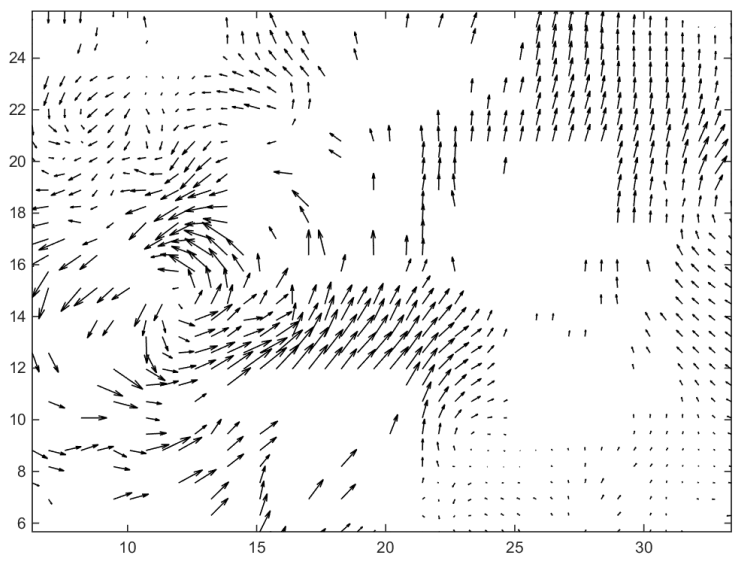

(b) Gappy field

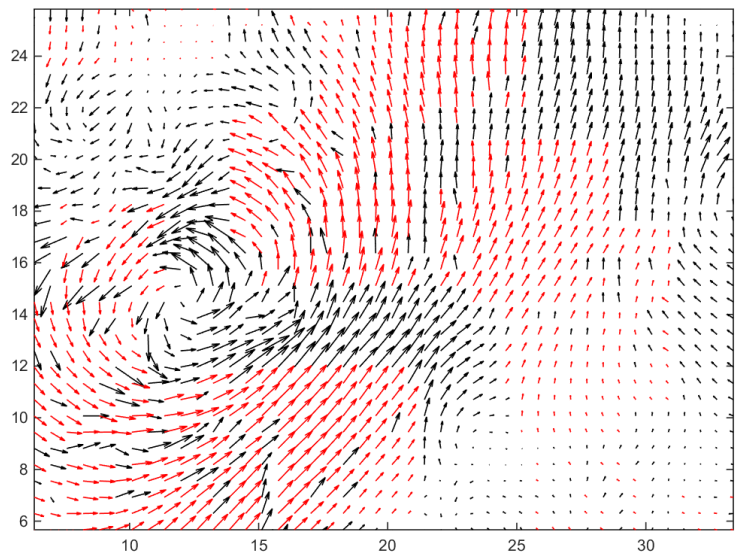

(d) GPOD reconstructed field after 10 main iterations

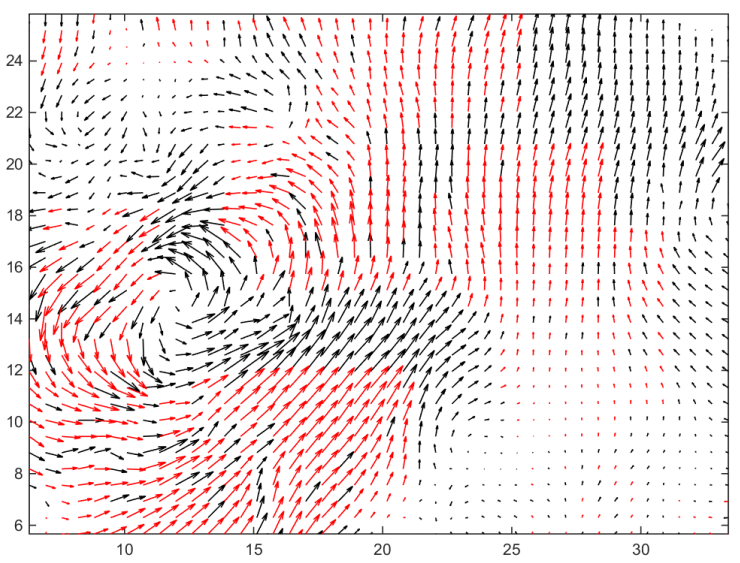

(e) GPOD reconstructed field at optimal number of modes

Figure 2: Zoomed in section of a single instantaneous velocity field reconstructed via the MF method. For this particular snapshot, the gappiness percentage is $45 \%$ and the reconstruction error in $u_{x}$ and $u_{y}$ is 0.454 and 0.447 , respectively, after 10 modes, and 0.226 and 0.231 , respectively, after the optimal number of modes $\left(\left.P^{*}\right|_{u_{x}}=526\right.$ and $\left.\left.P^{*}\right|_{u_{y}}=532\right)$. To make the visualization of the velocity vectors easier, the reconstructed vectors are shown in red and the third velocity component, $u_{z}$, has been omitted. Axes are measured in millimeters. 


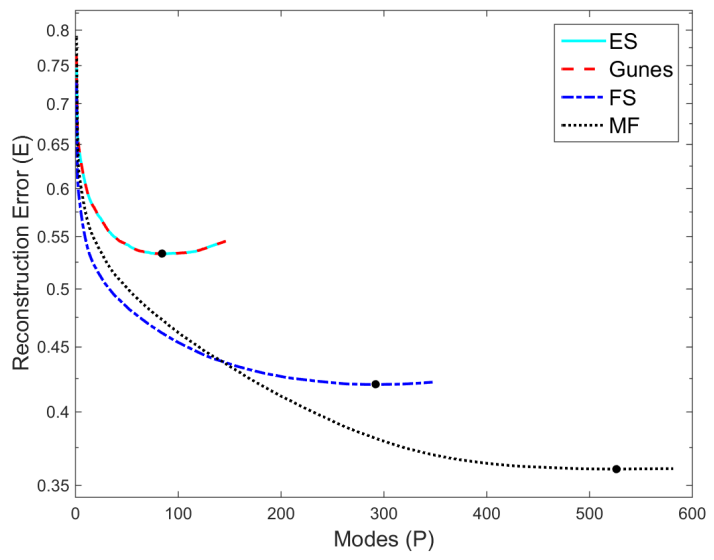

(a) $u_{x}$

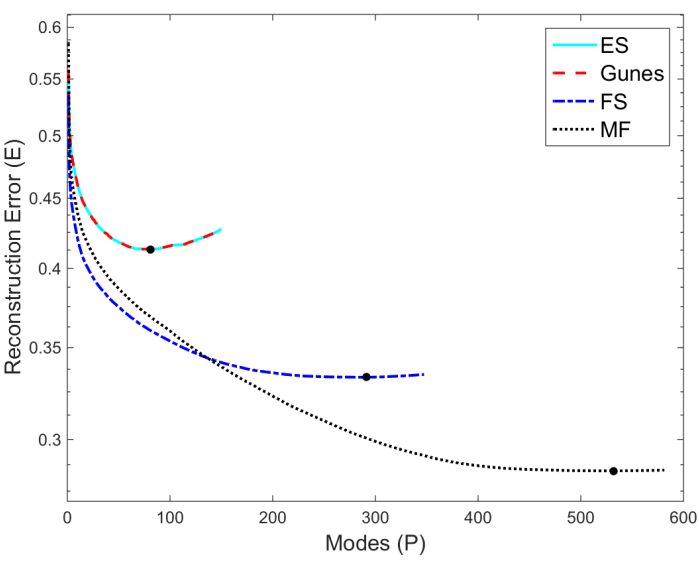

(b) $u_{y}$

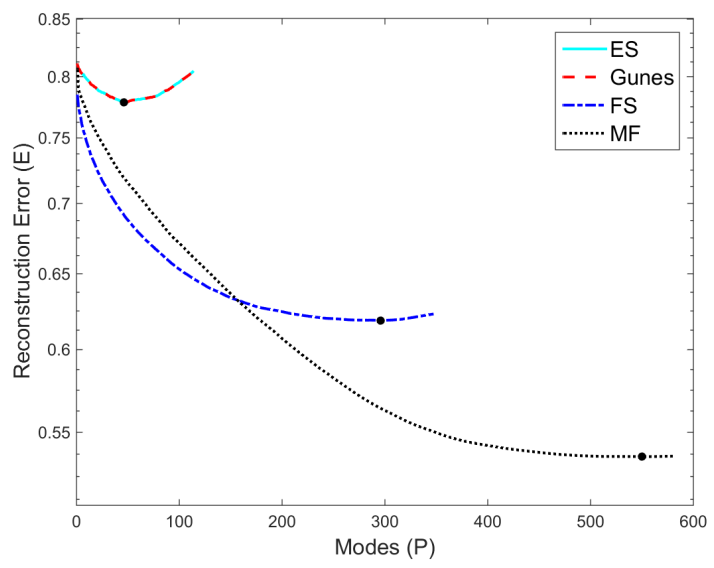

(c) $u_{z}$

Figure 3: Error curves of the four GPOD methods with $G=15 \%$. Black dots $\left(P^{*}\right)$ are at the error minima $\left(E^{*}\right)$ of the corresponding curves.

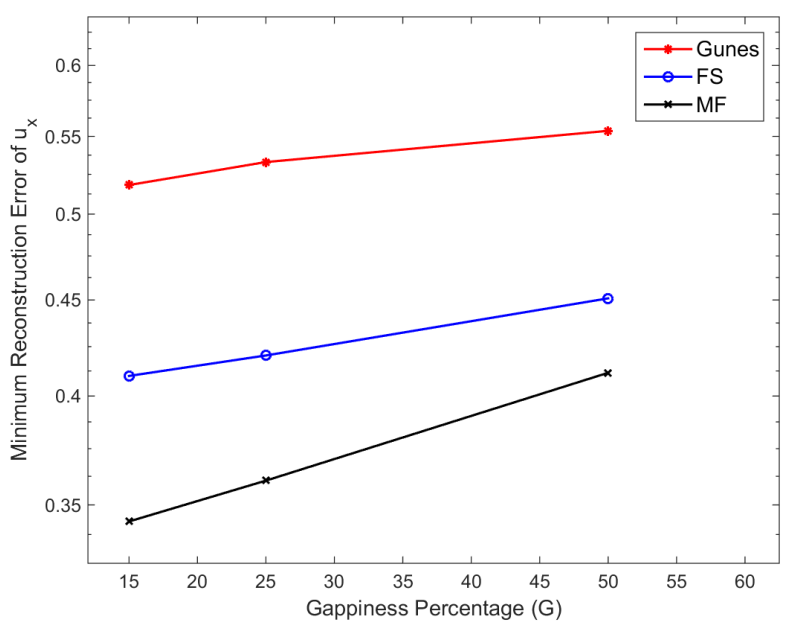

Figure 4: Minimum error values, $E^{*}$, of $u_{x}$ component as a function of gappiness percentage. 


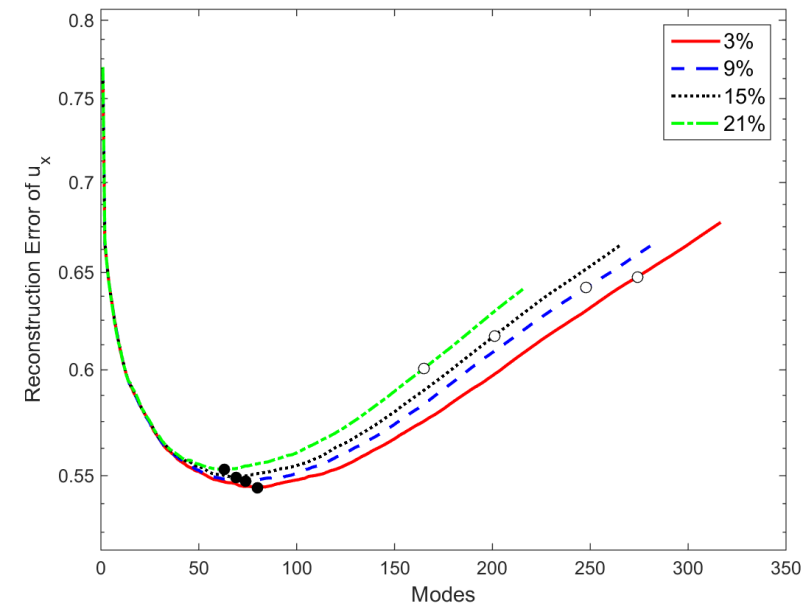

(a) Gunes method

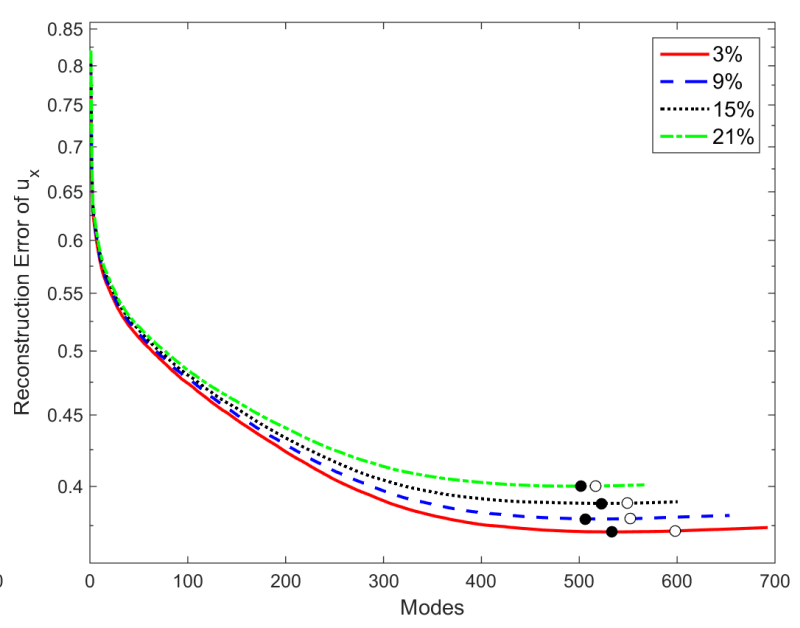

(b) MF method

Figure 5: Curves of $E$ for varying levels of CC gaps. Both the Gunes and MF method were run at 25\% gappiness of the 'real' gaps. Only $u_{x}$ has been shown. Black dots represent minima of $E$ while white dots represent locations of the minima of $E_{c}$.

low gappiness levels of the $\mathrm{CC}$ gaps, which prevents it from accurately reflecting the convergence of the real gaps. However, as the gappiness of the $\mathrm{CC}$ gaps increases, $P_{c c}^{*}$ begins to decrease while $P^{*}$ remains more or less the same. Therefore, for higher CC gaps' gappiness percentages, $P_{c c}^{*}$ acts as a reasonable convergence check, although it results in a higher $E$ as the overall gappiness percentage has been increased. Therefore, in order to implement this convergence check, GPOD needs to be run on a data set with a high number of $\mathrm{CC}$ gaps in order to determine $P_{c c}^{*}$. The reconstructed fields from this run are discarded as the error would too high given the high overall gappiness percentages. However, $P_{c c}^{*}$ can then be used to run GPOD again, now without any CC gaps, to get reconstructed fields with low error as there are no additional gaps and because the GPOD method has been stopped close to the optimal number of modes.

The above method also works well for the MF method, as shown in Figure 5b. Unlike the Gunes method, the MF method's $E$ curves are much flatter, and so the increase in error in $E$ between $P^{*}$ and $P_{c c}^{*}$ is visibly quite small. In the Gunes method, the error increase is $20 \%$ for the base case $(3 \% \mathrm{CC}$ gappiness) while for the MF method, the error increase is only $0.2 \%$. This feature means the MF method can be run with few CC gaps ( $3 \%$ gappiness) and only in one pass as there is not a significant decrease in error between $P^{*}$ and $P_{c c}^{*}$.

In the second convergence checking method, the turbulent kinetic energy $(K)$ is calculated from the eigenvalues of the POD approximation at each main iteration. Shown in Figure 6 are the $E$ curves of the Gunes and MF methods, run at $25 \%$ gappiness. Black dots on the curves are the minima of $E$ and the red dots are the maxima of $K\left(P_{K}^{*}\right)$. For the Gunes method, $P_{K}^{*}$ is much closer to $P^{*}$ than any of the $P_{c c}^{*}$ values from Figure 5a. This makes using turbulent kinetic energy a good measure of convergence when using the Gunes method. On the other hand, this technique does not work too well for the MF method, in terms of the spacing between $P^{*}$ and $P_{K}^{*}$, but since the error curve of the MF method is relatively flat near $P^{*}$, the increase in error is insignificant $(\approx 0.5 \%)$.

\section{Conclusion}

In this paper, GPOD was implemented, for the first time, on S-PIV measurements of a turbulent swirl flame typical of modern gas turbine combustors. Four different GPOD methods were studied: two non-adaptive (ES and Gunes) and two adaptive (FS and MF), with the last one being a new method. The analysis in this work consisted of adding varying amounts of artificial gaps to a non-gappy data set of an atmospheric pressure turbulent flame, running the aforementioned GPOD methods the subsequent gappy fields, and then comparing the resulting reconstructions to the original data. 


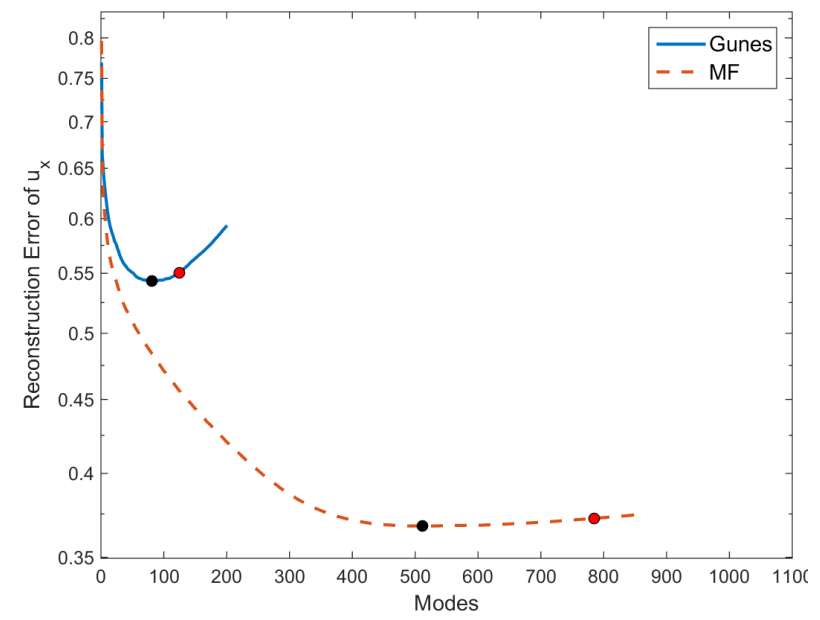

Figure 6: Plots of error curves of Gunes and MF methods run at 25\% gappiness. Black dots are minima of $E$ and red dots are the location of the maximum $K$.

From the results, the adaptive methods were found to be more accurate than the non-adaptive ones, with a difference in minimum error of over $20 \%$. The MF method was more accurate than all of the other methods; this held true for low and high gappiness percentages. It was also determined that the error curves (vs. main iterations) of all methods have a minimum point after which there is an increase in error. The location of the error minima varies with the velocity components, thereby indicating that a decoupled POD analysis, with respect to the velocity components, is required to minimize the reconstruction error.

In order to make the GPOD methods more applicable to real gappy data, two convergence techniques were analyzed. In the first technique, convergence was assessed from the minima of the reconstruction error calculated over artificially added CC gaps. It was determined that by adding a large number of CC gaps, close to the number of real gaps, a reasonable convergence check can be attained, albeit at the cost of increased reconstruction error given the increase in overall gappiness. Therefore, two passes of GPOD would be needed to implement this technique: one with large amounts of CC gaps, to determine the optimal mode, and the second with no CC gaps at all, to get a low error reconstruction stopped at the optimal number of modes. In the second convergence technique, the maxima of the kinetic energy was calculated from the POD eigenvalues. This technique provided a better convergence check for the Gunes method when compared to the $\mathrm{CC}$ gaps convergence technique. It performed comparatively poorly for the MF method, but it is still a viable convergence check since the curve of $E$ remains relatively flat for the MF method after its optimal number of modes.

\section{References}

1 Huang, Y. and Yang, V., "Dynamics and stability of lean-premixed swirl-stabilized combustion," Progress in Energy and Combustion Science, Vol. 35, No. 4, 2009, pp. 293-364.

2 Gunes, H., Sirisup, S., and Karniadakis, G. E., "Gappy data: To Krig or not to Krig?" Journal of Computational Physics, Vol. 212, No. 1, 2006, pp. 358-382.

3 Everson, R. and Sirovich, L., "Karhunen-Loeve procedure for gappy data," Journal of the Optical Society of America A, Optics and Image Science, Vol. 12, No. 8, 1995, pp. 1657-1664.

4 Raben, S. G., Charonko, J. J., and Vlachos, P. P., "Adaptive gappy proper orthogonal decomposition for particle image velocimetry data reconstruction," Measurement Science and Technology, Vol. 23, No. 2, 2012, pp. 025303.

5 Westerweel, J. and Scarano, F., "Universal outlier detection for PIV data," Experiments in Fluids, Vol. 39, No. 6, 2005, pp. 1096-1100. 
${ }^{6}$ Liang, Y., Lee, H., Lim, S., Lin, W., Lee, K., and Wu, C., "Proper orthogonal decomposition and its applications Part I: Theory," Journal of Sound and vibration, Vol. 252, No. 3, 2002, pp. 527-544.

7 Sirovich, L., "Turbulence and the dynamics of coherent structures. Part I: Coherent structures," Quarterly of Applied Mathematics, Vol. 45, No. 3, 1987, pp. 561-571.

8 Venturi, D. and Karniadakis, G. E., "Gappy data and reconstruction procedures for flow past a cylinder," Journal of Fluid Mechanics, Vol. 519, 2004, pp. 315-336.

9 Raffel, M., Willert, C. E., Wereley, S. T., and Kompenhans, J., Particle Image Velocimetry: A Practical Guide (Experimental Fluid Mechanics), Springer, 2007.

10 Steinberg, A., Arndt, C., and Meier, W., "Parametric study of vortex structures and their dynamics in swirl-stabilized combustion," Proceedings of the Combustion Institute, Vol. 34, No. 2, 2013, pp. 3117-3125.

11 Caux-Brisebois, V., Steinberg, A., Arndt, C., and Meier, W., "Thermo-acoustic velocity coupling in a swirl stabilized gas turbine model combustor," Combustion and Flame, Vol. 161, 2014, pp. 3166-3180. 